est considérable par rapport â l'effort retardateur provenant des résistances passives, les seules en jeu.

Il en résulte en pratique une très grande stabilité.

Ce redresseur est appelé aussi régulateur, parce qu'il permet, non seulement de transformer du courant alternatif en courant continu, mais aussi de régler à volonté la tonsion de ce courant continu tout en laissant constante la tension du courant alternatif reçu. Ce réglage se fait depuis zéro jusqu'au voltage maximum, sans perte d'énergie, par simple modification du calage des frotteurs servant à recueillir le courant continu sur la partie tournante de l'appareil.

Un appareil d'essai d'une puissance effective de 500 kilowatts a été construit, à la fin de l'année 1904, pour le compte de la Compagnie P.-L.-M., dans les ateliers Schneider et $\mathrm{C}^{1 e}$, à Champagne-sur-Seine, et a pu être soumis à une première série d'expériences et de mesures; en mai et avril 1905, dans la station centrale du Chemin de fer Métropolitain à Bercy, où le courant alternatif dont nous avions besoin a été mis à notre disposition.

Ces premiers essais, dont les résultats ont élé exposés en détail dans la Revue Générale des Chemins de fer et Iramuays (no doctobre 1905), ont permis de constater que le redressement et le réglage du courant se faisaient dans d'excellentes conditions et que la stabilité de l'appareil était parfaite.

La commutation était très satisfaisante et le rendement total, constaté par des mesures précises faites avec des appareils enregistreurs, dépassait, toutes pertes comprises, $88 \%$ pour un débit de $400 \mathrm{kw} ., 86 \%$ pour un débit de $200 \mathrm{kw}$, et $79 \%$ pour un débit de $100 \mathrm{kw}$.

Le rendement était d'ailleurs à peu près indépendant de la tension du courant continu fourni par l'appareil, ce qui montre que le réglage de la tension se faisait sans perte sensible d'énergie.

Une deuxième série d'essais vient d'être faite récemment avec le redresseur-régulateur par la Société d'Electricité Alioth, dans ses ateliers de Múnchenstein, près Bâle, en vue de compléter les essais que nous avions faits à Paris en 1905 et d'élucider certains points spéciaux qui avaient été primitivement laissés de côté.

On a vérifié ainsi qu'on pouvait sans aucune difficulté alimenter avec du courant redressé des moteurs à excitatation séparée, et qu'on pouvait aussi, à l'aide du redresseur-régulateur, transformer du courant continu à tension valiable en courant alternatif à tension constante.

Il résulte de ce qui précède qua, grâce à l'emploi des redresseurs-régulateurs. un pourra tacilement construire des locomotives électriques dans lesquelles la dépense d'énergie électrique será exactement proportionnée à la production du travail mécanique au moment des démarrages, et pendant la marche normale, et qui jouiront aussi de la propriété précieuse de restituer au réseau, sous forme de courant alternatif, l'énergie disponible correspondant à la diminution de force vive lors des ralentissements, ou au travail de la gravité pendant la descente des pentes.

En raison de l'extrême modérabilité des redresseursrégulateurs, on pourra régler la vitesse sur les pentes et ralentir les trains presque jusqu'à l'arrêt complet, sans faire usage des freins mécaniques.

En l'ésumé, en combinant les propriétés du courant continu avec celles du courant alternatif, il est aujourd'hui possible de construire des locomotives électriques d'une puissance considérable, plus grande que celle des plus fortes locomotives à vapeur; avec ces nouvelles locomotives, le coút de la traction électrique ne serait, dans beaucoup de cas, pas plus élevé que le coût de la traction à vapeur sur les profils faciles, et sur les profils accidentés, grâce à la récupération, il deviendrait très nettement inlérieur.

\section{TRACTION ÉLECTRIQUE DES BATEAUX}

Rapport de M. Léon Gérard

ingénieur, ancien président de la Société Belge des Electrictens.

\section{HISTORIQUE}

L'évolution des moyens de transports est un des faits les plus saillants de l'histoire contemporaine.

Cette évolulion s'est opérée d'une manière très curicuse, car elle a tour à tour fait abandonner et reprendre les divers Imodes usuels pour les perfectionner ensuite et les déveop per.

Dans les pays les plus industriels, la locomotive, aussitôt après son adoption, a fait complètement abandonner la route de terre, et le désir de limiter la concurrence des moyens de transport a poussé les puissantes compagnies de railways à accaparer les canaux pour les réduire à l'impuissance ; c'est l'histoire des canaux houillier's alimentant les ports anglais au début du XIX siècle.

La France a eu l heureuse fortune de n'avoir jamais abandonné ses admirables routes. Elle en est récompensće par l'essor si remarquable de sa grande industrie nationale de l'automobile.

Par un singulier retour des lois du progrès, toutes les perfections de la mécanique s'appliquent en ce moment à remettre en pleine activité le transport rapide sur route et bientôt sans doute le transport lourd.

Cette évolution récente est d'autant plus remarquable que l'on considérait, il n'y a pas vingt ans, le transport sul' route comme ne présentant qu'un mode arriéré de locomotion destiné à tomber en complète désuétude.

Le même phénomène s'est produit en matière de navigation intérieure. Là, encore, la France jouit à l'heure actuolle du remarquable avantage d'avoir sans cesse entretenu et perfectionné ses voies navigables, et, après une période d'erreur économique qui avait presque livré l'exploitation de ces voles a leur victorieux concurrent, le chemin de fer, on vit successivement se produire l'abolition des péages, la régularisation des grandes rivières, l'approfondissement et la systematisation d'un admirable réseau de canaux dont la législature a décidé, il y a quelques années, l'extension d'aplès un plan grandiose.

\section{IMPORTANCE ÉCONOMIQUE}

En 1905 , le trafic des quelques rivières canalisées françaises atteignait plus de 600 millions de tonnes kilomètres et 1470 kilomètres de canaux proprement dits assuraient à eux seuls un trafic de 2 milliards de tonnes kilométriques. Ces chiffres sont relatils à une petite partie seulement du réseau des canaux trançais, dont l'utilisation économique généralisée dépendra de divers facteurs, parmi lesquels le prix du halage et la vitesse sont des éléments essentiels. La France possédait, en 1904,8 325 kilómètres de rivières, canalisées en 4930 kilomètres de canaux.

Afí de se faire une idée comparative de l'importance économique des transports par eau, le fret moyen par eau pour les marchandises pondéreuses est, en France, le quart du prix du transport par rail.

En Allemagne, ce chiffre est du sixième. En Angleterre le transport par eau est dix fois moins cher et il est, en Belgique et en Amérique, six fois moins couteux que le transport par rail.

L'ensemble du Irafic par eau en France, que l'on peut estimer grossièrement à plus de 4 milliards de tonnes kilométriques, économise annuellement à l'industrie française plus de 96 millions de francs sur les dépenses de transport.

Les raisons techniques de celte formidable supériorité économique des transports par eau résultent de la réduction des efforts de traction, de la grandeur des unités de transport. L'unité moyenne est en France de 350 tonnes, et ce chiffre tend à grandir, et la barque type de navigation intérieure atteindra probablement 600 tonnes. 
Les conséquences de ila réduction des frais de transport sont considérables, je n'ai pas à en développer bien longuement les résultantes économiques. Les progrès si rapides et si décisif's de la grande industrie métallurgique ainéricaine, et spécialement celle des centres de l'Ouest, sont dûs à la création des grandes unités de transport à bon marché sur rail et de ces formidables unités de transport par eau qui sillonnent les lacs du Nord. Ces outils, qui permettent le transport des cokes et des minerais sur de très longues distances, à des prix dérisoires, cornparés à nos prix, sont la force industrielle de l'Amérique.

D'autres pays que la France etl'Amérique peuvent songer aussi à utiliser de très longs parcours pour de fortes charges transportées par unités de transports importantes. Je n’iusisterai pas sur l'importance des réseaux hollandals et belges (ce dernier, avec 1620 kilomètres, a un trafic de près de 1 milliard de tonnes kilométriques) parce qu'ils ne comportent que des trajets assez courts, mais je signale l'immense réseau des voies navigables de la Russie, dont $\mathrm{M}$. le professeur Merczyng a tracé un tableau si intéressant au dernier Congrès international de Navigation en $1905\left(^{*}\right)$.

Parmi ces voies, une série d'entre elles, allant de la Caspienne à la Baltique, a 4000 kilomètres de développement et forme une artère essentielle du trafic continental.

Le trajet d'Astrakan à St-Pétersbourg comportait en 1904, à lui seul, 5 millions de tonnes, formant près de 10 milliards de tonnes kilométriques.

Le réseau des canaux intérieurs, connu sous le nom de système Marie, comprend 1100 kilomètres de voies, avec un mouvement de 4 millions de tonnes. Ce réseau est en voie dextension et de réfection.

L'Allemagne, après avoir admirablement systématisé le cours de ses grands fleuves, dont elle fait des voies de transport à service intensif (le mouvement du Rhin attein à lui seul 5 milliards 300 millions de tonnes kilométriques), poursuit avec tenacité l'exécution du canal du Rhin-ElbeOder; quoiqu'elle dispose déjà, en plus de son important réseau fluvial, de 400 kilomètres de grands canaux ayant plus de $11 / 2$ millions de tonnes trafic, et que son réseau canalisé soit de 2530 kilomètres.

L'Angleterre a laissé ensabler et tomber en désuétude plus de 5500 kilomètres de canaux dont les proportions et le tirant d'eau ne sont plus appropriés aux exigences du trafic moderne.

L'opinion publique, les économistes, l'élite scientifique et technique, ont porté récemment leur attention sur cet état de choses, et sans doute l'esprit pratique et énergique des Angrlais trouvera une solution à ces difficultés précisément dans l'adoption des moyens mécaniques et de la traction électrique.

Le grand développement du trafic des voies navigables continentales est dù surtout à la dimension des unités de transport sur les rivières canalisées.

La généralisation des moyens mécaniques, la substitution au halage animal de procédés mécaniques, la substitution de tarifs réguliers aux marchandages des frets, l'organisation des transports à heure fixe et leur systématisation sont autant de mesures qui abaisseront encore le prix des transports pai eau et assureront avec une plus grande régularité un effet utile plus considérable du réseau exploité.

Si l'on songe qu'une réduction de chaque millime de fret pour chaque milliard de tonnes kilomètres portées par voie de canaux représente un bénéfice d'un million de francs; si l'on considère que les canaux ne transportent pas actuellement la dixième partie du tonnage qui représenterait leur capacité réelle, on peut mesurer l'importance économique

(*) Associalion Internationale permanente des Congrès de navigation. X Congrès. Milan 1905, Etude économique sur la traclion mécanique des baleaux par H. Merczyng, ingénieur des voies de communication,professeur à l'Institut Impérial. considérable du problème de' la traction mécanique des bateaux au point de vue social.

\section{Procédés de Halage et de Touage}

Les procédés mécaniques du transport sur canaux sont des procédés de remorquage par hélice, de touage par câble mobile ou par câble ou chaîne immergés, ou de halage par tracteur circulant sur la berge.

Disons brièvernent que les ingênieurs français ont joué un rôle prépondérant dans ces recherches, avec les Améri. cains et les Belges.

Rappelons brièvement les noms de Levy, de De Mas, de La Rivière, Bourguin, Galliot, de Bovet, Lombard-Gérin, qui, avec tant d'autres Français, ont étudié pratiquement ces questions. Rappelons que c'est un ingénieur belge, Bouquié, qui a réalisé le premier touage à vapeur sur chalne immergée, et que les Américains ont été les pionniers de l'emploi de l'électricité à la propulsion des bateaux, pour passer à l'examen de l'état actuel des recherches relatives à la traction des bateaux par les procédés électriques.

\section{Supériorité des Procédés ÉLEctriques}

Les procédés de halage et de touage électriques tirent leur supériorité des mêmes causes qui ont déterminé l'em. ploi de l'électricité dans les autres domaines de la traction.

Le haut rendement des petites unités électriques, la divisibilité de la puissance, la légèreté des moteurs, la simpli. cité de leur maniement, la rapide accélération des mobiles, la facile distribution à distance d'une puissance d'autant plus économique que sa production est plus centralisée sont autant de facteurs généraux de cette supériorité.

Parmi les avantages spéciaux aux procédés électriques au point de vue de la navigation intérieure, on doit ranger en première ligne l'ahsence des remous qui rendent l'empló des remorqueurs à hélice très onéreux par la destruclion des berges, la facilité du démarrage progressil, le petit encombrement des appareils et leur docilité, leur haut ren. dement mécanique, l'absence de consommation au repos, la rapide motion des appareils à vide, la faible main-d'cuvre exigée pour leur maniement, la possibilité propre à certains systèmes de s'appliquer à la voie sans modifier les ouvrages d'art existants.

L'électricité permet le rapide maniement des écluses el des ponts tournants, l'alimentation artificielle des biefs et l'éclairage de la voie.

Les principaux procédés de traction électrique sont:

L.e halage sur berge sans rails (type Gaillot).

Le halage sur berge à crémaillèré (type Siemens).

Le halage sur berge sur voie bi-rail lisse (types de Gérard, Chanay el Siemers).

Le halage sur berge sur voie monorail lisse à adhérence proportionnelle (système américain type Wood-Gérard).

Le touage électrique ou mécanique sur chaîne à adhérence magnétique (système de Bovet).

Le touage électrique sur câble.

\section{Traction sur Berges sans RaIls}

Après une longue expérience, fournie par l'exploitation des canaux français et belges, on peut considérer la traction sur berge sans rails comme un système condamné par l'ex. périence, encore que son application puisse être fort. utlle pour des services temporaires ou spéciaux.

Le côté séduisant du système, qui est de n'exiger aucune installation de voie, est compensé par la nécessité de dis: poser d'une chaussée fortement empierrée dont le coût est considérable et dont lentretien a atteint en France annuel. lement $875 \mathrm{fr}$. par kilomètre (*) et $1900 \mathrm{fr}$. en Belgique.

(") Association internationale permanente des congrès de navigation. 10. Congrès. Milan. Etude économique et technique de la traction mén: 
D'autre part, l'élévation du prix d'entretien du matériel cahıté dans les ornières de la route, et le rendement faible des appareils ont prouvé, après l'expérience française et belge, l'économie à résulter de l'emploi du rail.

\section{Traction sur RAIL a CRÉmaIltère}

La maison Siemens, de Berlin, établit un système de ce genre, basant l'adhérence du tracteur d'abord sur la crémaillère (Canal de Finow), puis sur l'emploi d'un rail guide, la roue motrice trouvant son adhérence sur le gravier de la route empierrée. Ces deux systèmes présentaient des défauts de prix et des difficultés tels que leurs applications furent des plus réduites et que ses inventeurs, aprés la publication au Congrès de Dusseldorf (1902), des travaux belges et francais sur l'emploi des rails lisses, et la détermination des efforts de démarrage et de traction des bateaux (travaux de M. Mollard, ingénieur à Paris et rapport cité plus haut *), appliquèrent sur deux kilomètres, au canal Teltow, en 1905, les systèmes expérimentés presque en même temps en 1902 el aussitôt appliqués au canal d'Aire et de la Deule, en France, et au canal de Charleroi, en Belgique, c'est-à-dire le système à voie bi-rail lisse en abaudonnant complètement la voie à crémaillère.

\section{TRACTIÓN SUR VOIE BI-RAIL LISSE}

Jusqu'en 1901, l'on admettait, au point de vue scientifique, que les efforts de démarrage d'un bateau en écluse, ou en profil de section réduite en canal, étaient de si grande valeur qu'aucun engin à adhérence simple n'était capable d'entrainer un bateau.

L'étude analytique et expérimentale du phénomène de démarrage me convainquit de la possibilité de résoudre la la question, sans exagération du poids du véhicule, par un réglage approprié des résistances du moteur dont la courbe d'accélération devait présenter un caractère en rapport avec le rapport des profils mouillés et du poids du bateau.

L'expérience conduisait, à peu de temps de là, les ingénieurs français Mollard et Chanay à réaliser le démarrage sur rails lisses à l'aide dune locomotive minière symétrique lestee d'une charge de fonte brute.

Dès lors, la supériorité de la traction sur rails lisses, non pas au point de vue du coût du premier établissement, mais au point de vue du prix d'exploitation (consommation de courant, entretien de la voie, du matériel et amortissement) fut reconnue.

C'est sur la base de ce système, rẻalisé en principe dès 1902, que les 83 kilomètres formant le réseau des canaux d'Aire, de la Deule et de la Scarpe en France furent projetés, et que la traction sur rail lisse s'étendit progressivement sur les 58 kilomètres déjà concédés depuis 1889 .

En Belgique, l'absence de disposition légale sur la concession à longs termes des chemins formant les berges, pour permettre la construction des voies permanentes métalliques, et l'impossibilité pour les exploitants d'avoir cependant un temps de concession sulfisant pour permettre lamortissement du capital investi dans les voies, fit ajournor de manière indéterminée l'établissement du sytème. Cette détermination était d'autant plus nécessaire que le canal de Charleroi étant en voie d'élargissement, ses berges devaient subir pendant quelques années de nombreuses modifications.

En Allemagne, le concours établi en 1903 par l'Etat prussien pour l'èlectrification du canal de Teltow avait primé le système Siemens de Finow, lequel ne fut cependant pas appliqué.

nique des bateaux sur les fleuves, canaux et lacs. Rapport de M. M.-G. La Riviere, ingénieur des ponts et chaussées, à Lille.

(*) 9* Congrès international de navigation. Dusseldorf 1902. Traction électrıque des bateaux. Détermination des efforts de démarrage et de traction, par Léon Gérard, ingénieur, président de la Société Belge des Electricions.
Les études de la direction du canal de Teltow conduisirent au choix, pour une partie du canal, d'un système de bateau remorqueur à hélice actionné soit par accumulateurs, soit par trolley, avec trolley auto-moteur Lombard-Gérin. Le rendement d'une hélice de touage dont le glissement est considérable, combiné avec le rendement des accumulateurs ne devait pas amener à des résultals fort économiques, et l'adoption de ce système ne devait évidemment avoir qu'un pur intérêt de recherche.

D'autre part, pour deux ou trois kilomètres de traction sur berge, on adopta un système de locomolive dyssimétrique à six roues, comprenant quatre moteurs, dont deux pour actionner les roues, l'un pour relever et abaisser un martereau de halage, et le quatrième pour actionner le treuil d'enroulement emprunté au tracteur belge du type de 1899. Ce système ne se distingue guère du système de locomotive française que par la complication de ses organes, et le grand encombrement du système qui représente une compilation fort heureuse des procédés employés jusque-là en Europe.

L'encombrement de l'appareil inauguré avec quelque solennité au canal de Teltow, en 1906, est de $6,80^{\mathrm{m}} \times 1,80^{\mathrm{m}} \times$ $2,80^{\mathrm{m}}$, le poids de la locomotive est denviron 11 tonnes. Son rendement moyen au crochet est en moyenne $65 \%$ avec une puissance normale de $16 \mathrm{HP}$. Le service projeté pour la traction du canal Teltow devra porter sur 37 kilomètres.

La locomotive française de halage, employée au canal du Nord depuis 1903, pour un réseau de 83 lílomètres, dont 58 sont actuellement en exploitation, a $4,80^{\mathrm{m}} \times 1,40 \mathrm{~m} \times 2,50 \mathrm{~m}$ et pèse seulement 8 tonnes: son rendement moyen est de $671 / 2 \%$ avec une puissance maxima de 40 chevaux pour une puissance normale de $20 \mathrm{HP}$.

A la différence de la locomotive allemande, dont six dixièmes seulement du poids mort est adhérent, et dont la dyssimétrie ne permet que le halage dans un sens, la locomotive française est symétrique, à adhérence à poids total, et fonctionne dans les deux sens avec facilité. Ce fait permet le service à une voie pour les canaux dimportance moyenne.

Tandis que les expériences allemandes et les exploitalious françaises se poursuivaient sur la base du système bi rail, réalisé d'abord en Belgique, les Américains réussissaient à mettre en usage, en matièrc de halage électrique, lo système monorail déjà inutilement tenté en Europe par Rudolph et Feldmann.

Les premiers appareils de Wood, expérimentés en 1903 sur le canal Erié, se réduisaient aux moteurs, avec leurs engrenages. circulant sur des poutres à double té, lesquelles étaient pincées verticalement par des galets à ressort. L'exagération des vitesses pratiquées $(9$ à 10 kilomètros à l'heurc), la grandeur des unités remorquées, empêchèrent de tirer de ce système tous les avaritages résultant de soll haut rendement, de sa compacité et de l'absence de tout poids mort.

Dès 1905, les recherches simultanées de Mr St John Clarke, ingénieur en chel du Métropolitain de New-York, de Mi. Francis Blackwell, et de moi même, conduisirent à la réalisation d'un type d'appareil monorail caractérisé par les deux faits que l'adhérence d'un appareil de moins de 3 tonnes permettait de développer des efforts au crochet de 4000 kilogrammes, et que le rendement moyen de l'appareil était de $80 \%$ pour une puissance normale de $45 \mathrm{HP}$. L'en. combrement de l'appareil est de 1,65 × $0,75 \times 1,65$.

La voie consiste en une simple poutrelle double té du commerce, pesant 37 kilogrammes par mètre.

Le même appareil réduit, dans le but d'application a la plupart des canaux de dimensions moyennes en Europe, a 14 chevaux et mesure $1,40 \times 0,45 \times 1,25$. La poutre servant de voie est réduite à un double té de 175 millimètres pesant 21 kilogrammes par mètre.

En comparant le système américain, à adhérence propor- 
tionnelle, au système européen, le poids mort des appareils atteint seulement le quart des appareils à adhërence simple et le rendement net est de $15 \%$ supérieur.

Du fait de ses faibles dimensions, l'appareil est le seul pouvant donner en tunnel un appareil de grande puissance, n'encombrant pas le débouché par ses voies et sun gabarit propre. En canal courant, nulle occupation du chemin de halage qui reste libre; sous les ouvrages d'art, nulle nécessité d'augmentation de portée; sur les quais, la voie s'applique comme une simple main courante sur la paroi verticale des parapets, ou sur une voie surélevée au-dessus des bateaux en déchargement $\left(^{\star}\right)$.

\section{TOUAGE Électrique SUR CHAINE}

Le touage électrique sur chaine jouit des excellents caractères économiques du touage à vapeur sur chaine. Ses applications sont limitées aux canaux à fort trafic et à longs biefs; et le prix élevé du premier établissement de la chaine, l'usure de ses maillons sur les roues à empreintes, ont causé souvent des déceptions que l'application ultérieure de l'électricité n'est pas parvenue à réparer complètement. L'abandon des roues à empreintes et la substitution des poulies magnétiques de De Bovet atténuent le dernier de ces défauts autant pour les touages à vapeur que pour les touages sur chaines.

\section{Touage Électrique sur Cable}

Pour les petits canaux à faible trafic, et là où la présence de bateaux toueurs ne constitue pas une gêne de service, le système du touage sur câble peut produire d'excellents résultats, à condition de ne devoir exercer que de faibles efforts de traction dans des canaux à rayons suffisamment glands.

Dans ces appareils, l'adoption du principe d'adhérence employé en Amérique conduit aux mêmes résultats d'effi cacité, de rendement et à la réduction dans une mesure considérable du capital de premier établissement à investir pour la voie.

Pour un tel système, la voie se réduit à un câble souple dont la valeur ne dépasse guère 1500 francs par kilomètre. Il en résulte que la possibilité d'application de la traction électrique au halage est étendue, par le fait de l'emploi d'un tel système, à des canaux de petit trafic qu'il était impossible de considérer jusqu'à présent comme exploitable mécaniquement.

Une étude économique détaillée du problème de l'électrification des canaux, électrification dont les conséquences directes et indirectes sont considérables pour l'exploitation du canal lui-même, et pour la région industrielle desservie par ce canal, ne peut trouver place dans les limites de ce rapport déjà trop long.

\section{CONCLUSIONS}

Il est utile de fixer en termes généraux que, dans l'état présent de la question, en usant de tarifs appropriés aux nécessités commerciales en France, le système allemand du canal de Teltow ne paraît guère applicable en raison de son prix, de sa complication et de son encombrement; que le halage sur rails doubles, tel qu'il est pratiqué dans le Nord, permet l'exploitation de canaux ayant plus de 2000000 de tonnes avec la certitude de rémunérer les capitaux engagés, et que ce système a donné des preuves pratiques d'application depuis cinq ans.

Que la traction sur monorail américain permet l'exploita. tion économique à tarif modéré de tout canal ayaint environ

(') Association internationale permanente des congrès de navigation. 10 Congrès. Milan 1905. Etudé éconornique et technique de la traction mécanique des bateaux sur les fleuves, canaux et lacs. Rapport de $M$. M. St-John Clarke, ingémeur conseil à New-York, et Léon Gérard, ingénieur, ancien président de la Société Belge dos Électriciens
1500000 tonnes de trafic, et que Ia simplicité du système,et son petit encombrement,en font le système par excellence, adaptable en souterrain et sous les ouvrages d'art; enfin que le touage sur câble, basé sur les mêmes principes, mais réduit à des unités de petites forces, permet d'appliquer les avantages de la traction électrique à des canaux ayant au plus 400000 tonnes de trafic ( $\left.{ }^{*}\right)$.

Ces données sont évidemment à corriger en fonction des tarifs et en fonction des conditions locales d'établissement et d'exploitation, mais il y a lieu de retenir ce fait que la technique des canaux dispose actuellement de moyens mó caniques permettant l'organisation systématique du halage, la substitution avantageuse de la machine à l'animal de trait, et que cet immense progrès a les conséquences industrielles les plus importantes au point de vue du grand problème économique des moyens de transport,

Les avantages économiques généraux à retirer de l'existence des lignes de distribution de force établies le long d'un canal, pour les populations, ne sont pas moindres:

La diminution des frets, l'augmentation du trafic du canal, la rapidité des échanges, favorisent le développement d'industries nouvelles le long de la voie navigable. Ces industries utilisent les lignes de force'préexistantes, et par une répercussion économique bien connue, le prix de l'électricité sur de tels réseaux tend à diminuer par la meil. leure utilisation des puissances installées.

L'importance économique de l'établissement du halage éloctrique est donc considérable, puisqu'il tend à faciliter l'établissemont de centres de production électrique à bon marché, au bénéfice de la généralité.

\section{APPLICATION DES TURBINES A VAPEUR AUX STATIONS CENTRALES D'ÉLECTRICITÉ}

\author{
Rapport de M. de Marchena \\ ingénieur en chef de la Compagnie Thomson-Houston
}

L'application des turbines à vapeur à la commande direcle des dynamos ne date vraiment que de quelques années; mais, dans ce très court laps de temps, elle a pris un dévé. loppement extraordinaire qui constitue certainement le fait le plus saillant de l'industrie électrique durant cette période.

A l'heure actuelle, pour ne citer que les types les plus en vogue, il y a en exploitation, ou en cours de construction environ:

600000 kilowatts en turbines Parsons;

600000 kilowatts en turbines Curtis;

100000 kilowatts en turbines Zoelly;

et cependant ces deux derniers types sont relativement récents, puisqu'elles n'ont paru sur le marché: les turbines Curtis qu'en 1902, et les turbines Zoelly qu'en 1903.

Le mouvement en faveur des turbines à vapeur est si général, et leur's avantages sont si universellement reconnus, èt hors de toute discussion, 'qu'à l'heure actuelle on ne concevrait plus guère de grande station génératrice constituée autrement qu'avec ces appareils.

Le principale difficulté qu'a rencontrée, à ses débuts, la turbine à vapeur résidait dans les vitesses élevées de rotation auxquelles leur emploi conduisait. Cette difficulté a été résolue par l'emploi des expansions plus ou moins. multipliées, permettant de réduire considérablement leśs. vitesses d'écoulement de la vapeur, et par suite les vilesses périphériques qui leur sont étroitement liées.

Ces expansions sont surtout nombreuses dans les types de turbines plus anciens, tels que les turbines Parsongs, dans lesquelles ont est ainsi arrivé à réduire les vitessies

(*) Ces chiffres sont établis en admettant pour le kulowalt-hrured prix de 0 fr. 10. 